\title{
Ultra-fast, sensitive and quantitative on-chip detection of group B streptococci in clinical samples
}

Qing Caia ${ }^{a}$, Maarten Fauvart ${ }^{a}$, Rodrigo Sergio Wiederkehra, Benjamin Jones ${ }^{\mathrm{a}}$, Piet Cools ${ }^{\mathrm{b}}$, Peter Goos $^{\mathrm{c}, \mathrm{d}}$, Mario Vaneechoutte ${ }^{\mathrm{b}}$, and Tim Stakenborg ${ }^{\mathrm{a}, \#}$

aimec, Kapeldreef 75, B-3001 Leuven, Belgium

${ }^{b}$ Laboratory for Bacteriology Research, Department of Clinical Chemistry, Microbiology and Immunology, Ghent University, Heymanslaan 10 185, Entrance 38 (MRB2), 9000 Gent, Belgium ${ }^{c}$ Division of Mechatronics, Biostatistics and Sensors (MeBioS), KU Leuven Kasteelpark Arenberg 30 - bus 2456, 3001 Leuven

${ }^{d}$ Department of Engineering Management, University of Antwerp, 2000 Antwerpen, Belgium

Running head: Ultrafast qPCR detection of GBS in patient samples

\#Corresponding author: Tim Stakenborg, Kapeldreef 75, B-3001 Leuven, Belgium, +3216281654, tim.stakenborg@imec.be

Keywords: ultra-fast, qPCR, clinical samples, on-chip, silicon, Streptococcus agalactiae

List of abbreviations: UF-qPCR, ultra-fast qPCR; GBS, group B streptococci; NTC, no template control; Ct, cycle threshold 


\section{Abstract}

PCR enables sensitive and specific detection of infectious disease agents, but application in pointof-care diagnostic testing remains scarce. A compact tool that runs PCR assays in less than a few minutes and that relies on mass-producible, disposable reactors could revolutionize while-you-wait molecular testing. We here exploit well-established semiconductor manufacturing processes to produce silicon ultra-fast quantitative PCR (UF-qPCR) chips that can run PCR protocols with limited assay optimization. A total of 110 clinical samples were analyzed for the detection of group B streptococci using both a validated benchtop and an on-chip qPCR assay. For the on-chip assay, the total reaction time was reduced after optimization to less than $5 \mathrm{~min}$. The standard curve, spanning a concentration range of 5 log units, yielded a PCR efficiency of $94 \%$. The sensitivity obtained was 96\% (96/100; $\mathrm{Cl}: 90-98 \%)$ and the specificity 70\% (7/10; $\mathrm{Cl}: 40-90 \%)$. As shown, in case melting analyses would be integrated, the obtained sensitivity would slightly drop to $93 \%$ (Cl: $86 \%-96 \%$ ), while the specificity would increase to $100 \%$ (Cl: $72 \%-100 \%)$. In comparison to the benchtop reference qPCR assay performed on a LightCycler ${ }^{\odot} 96$, the on-chip assay demonstrated a highly significant qualitative (Spearman's rank correlation) and quantitative (linear regression) correlation. Using a mass-producible qPCR chip and limited assay optimization, we were able to develop a validated qPCR protocol that can be carried out in less than five minutes. The analytical performance of the microchip-based UF-qPCR system was shown to match that of a benchtop assay. This is the first report to provide UF-qPCR validation using clinical samples. We demonstrate that qPCR-based while-you-wait testing is feasible without jeopardizing assay performance. 


\section{Introduction}

The diagnosis of microbial infections traditionally relies on culture based techniques often followed by biochemical or antimicrobial susceptibility tests to direct clinical decisions [1]. More recently, nucleic acid test after enrichment may be used $[2,3]$. Especially for cases where rapid screening or treatment is needed, nucleic acid based tests have moved into the clinical laboratories for detection of agents causing infectious diseases, increasingly replacing classical culture-based microbiology techniques. Recent developments, which integrate sample preparation and PCR in a single automated workflow with limited hands-on time like Xpert ${ }^{\circledR}$ from Cepheid, go one step further, providing access to molecular testing at the point of care. Still, turnaround times of typically 1 hour mean that true while-you-wait testing remains beyond reach. With techniques available that perform nucleic acid extraction in less than $10 \mathrm{~min}$, ultra-fast (in under $5 \mathrm{~min}$ ) quantitative PCR (UF-qPCR) is a target to effectively allow while-you-wait testing [4-6]. As noted previously [7], the present challenge for decreasing PCR amplification times lies with instrumentation, not with PCR chemistry. A substantial R\&D effort is spent investigating technologies that can reduce reaction times, specifically by speeding up the thermal cycling that is fundamental to PCR. Examples include using an oscillating fluid plug [8], continuous flow-through [9-12], static reaction mixtures [13-15] or liquidbased thermalization [16,17], resulting in PCR times down to 15 seconds [5]. In these different reports, the described technologies have either not been characterized intensively for analytical performance $[8,10,11,14-16]$, or sacrifice assay performance for speed $[9,12,13,17]$, while none provide clinical data to support their usefulness in a healthcare setting. Most importantly, none of the solutions described to date offer the perspective of integration into a compact tool that relies on mass-producible, disposable PCR reactors.

In this article, we describe the development, application and validation of silicon UF-qPCR chips produced using industry-standard semiconductor device fabrication processes, allowing easy upscaling to robust mass production. We used the chips to run a previously validated PCR assay for the detection of Streptococcus agalactiae (Group B streptococci, GBS). This target was selected 
because a molecular bedside test for GBS, which is harmful for newborn health when transmitted from the mother during childbirth, would significantly improve current screening strategies during antenatal care. Current screening for GBS is most often performed during late pregnancy (i.e., at 35 weeks of gestation), and women found positive receive antibiotic prophylaxis when in labor. However, due to the specific dynamics of GBS colonization (which appear or disappear on a day time), and not due to the diagnostic tool used, many women receive antibiotics unnecessary, when GBS was present at time of screening but no longer at delivery. Also, many women don't receive the necessary prophylaxis, because GBS is present at the time of delivery while it was not present at the time of screening. Therefore, a true improvement would be a diagnostic tool that allows screening when women come in the hospital for delivery. GBS detection therefore makes an excellent case for point-of-care DNA-based diagnostics and motivated the transfer of the assay to a fast chip format. We successfully analyzed 110 clinical samples on chip. After limited assay optimization, we obtained a high sensitivity and specificity confirming the strong agreement between the benchtop reference qPCR assay and the chip-based UF-qPCR assay performed in under 5 minutes per sample.

\section{Materials and methods}

\section{CLINICAL SAMPLES}

Samples were obtained in a previous study [2]. One-hundred women at 35 to 37 weeks of gestation, attending the prenatal clinic at Ghent University Hospital, Ghent, Belgium (with an average of 1,200 deliveries per year), were enrolled in the study from June 2009 to January 2010 . The study was approved by the Ethics Committee (IRB protocol nr. 2007/096) of the Ghent University Hospital, Ghent, Belgium. All women provided informed consent prior to collection of samples.

Rectovaginal, vaginal and rectal samples were collected using nylon flocked swabs that were submerged into $1 \mathrm{~mL}$ of ESwab transport medium (ESwab, Copan Diagnostics, Brescia, Italy). Volumes of $200 \mu \mathrm{L}$ from the ESwab transport medium of the rectovaginal ESwabs were inoculated 
into separate tubes with $5 \mathrm{~mL}$ of Todd-Hewitt broth with $1 \%$ yeast extract, $15 \mu \mathrm{g} / \mathrm{mL}$ nalidixic acid and $10 \mu \mathrm{g}$ colistin/mL (Lim broth, Becton Dickinson, Erembodegem, Belgium). The tubes were incubated aerobically overnight at $37^{\circ} \mathrm{C}$. DNA was extracted as described previously [2] and stored at $-20^{\circ} \mathrm{C}$ until use.

\section{SILICON CHIP FABRICATION}

The silicon chip (see Figure 1A) was fabricated as described by Majeed and colleagues [18]. In summary, the processing of chips is based on etching the fluidic structures in silicon, sealing them by anodic bonding with a Pyrex wafer and opening access holes from the silicon backside. The qPCR reaction chambers on the silicon chip have a nominal depth of $250 \mu \mathrm{m}$, a width of $500 \mu \mathrm{m}$ and a serpentine-shape design to overcome air trapping (Figure 1B) resulting in a total reactor volume of $2.4 \mu \mathrm{l}$. Chips had multiple PCR cavities depending upon design (Figure 1A), but only a single cavity was used for each PCR tests. Insulating trenches were etched around the reaction chamber (Figure 1A) to avoid undesirable heating of the bulk of the chip during thermal cycling.

\section{EXPERIMENTAL SETUP}

The silicon chip was mounted with a thermo-electric cooler (\#MPC701, Micropelt, Umkirch, Germany) for heating and cooling. A K-type thermocouple (\#CHAL-010, Omega, Norwalk, USA), fabricated from a $25 \mu \mathrm{m}$ diameter wire, was glued to a heat spreader for temperature monitoring (Figure S1A). The thermo-electric cooler and heat spreader were stacked and glued together to a heat sink (Figure S1A and S1B). A closed-loop PID controller with H-bridge switch (FTC200, Accuthermo Technology, Fremont, USA) was used to configure and to control the thermal cycling of the silicon chip. The Pyrex bonded to the silicon chip allows real-time fluorescence monitoring of the reaction chamber during thermal cycling using an inverted microscope (IX71, Olympus, Tokio, Japan) equipped with a CMOS camera (Orca Flash 4.0, Hamamatsu, Hamamatsu city, Japan) and fluorescent light source (X-Cite exacte, Excelitas Technologies, Waltham, USA).

PCR PROTOCOLS 
The S. agalactiae sip gene encoding the surface immunogenic protein was chosen as a qPCR target, generating a 78-bp long fragment, as described before [2].

Benchtop reference qPCR assay (performed with hydrolysis probe)

The benchtop reference qPCR assay was performed as before with minor modifications [2]. In short, we used the LightCycler ${ }^{\odot}$ Probes Master kit (Roche, Basel, Switzerland) combined with sip primers (500 nM each) and sip probe (200 nM) and ran the assay on the LightCycler ${ }^{\oplus 96}$ (Roche, Basel, Switzerland) in a total volume of $10 \mu \mathrm{L}$. Cycling conditions for the LightCycler ${ }^{\odot} 96$ were: $95^{\circ} \mathrm{C}$ for 5 min, followed by 40 cycles of $95^{\circ} \mathrm{C}$ for $10 \mathrm{~s}, 58^{\circ} \mathrm{C}$ for $15 \mathrm{~s}$ and $72^{\circ} \mathrm{C}$ for $20 \mathrm{~s}$. The total run time including ramp rates was $1 \mathrm{~h} 20 \mathrm{~min}$.

Standard on-chip qPCR assay (performed with an intercalating dye)

For the standard on-chip qPCR assay, a master mix with a total volume of $2.4 \mu \mathrm{L}$ was prepared containing 1x KAPA2G M master mix (Kapa Biosystems, Wilmington, USA), $250 \mathrm{nM}$ of sip primers, $2 \mathrm{mM}$ of dNTPs (Invitrogen, Carlsbad, USA), $1 \times$ EvaGreen (Biotium, Fremont, USA), $2 \mathrm{mM}$ of $\mathrm{MgCl}_{2}$ (Invitrogen Carlsbad, USA), $1 \%$ sulfolane (Sigma, Missouri, USA), $0.5 \mathrm{mg} / \mathrm{mL}$ ultrapure bovine serum albumin (Ambion, Foster city, USA), and 0.02 units $/ \mu \mathrm{L}$ of the $4: 1$ mix of KAPA2G fast polymerase and KAPA2G hot-start fast polymerase (Kapa Biosystems, Wilmington, USA). Following cycling conditions were used: 1 min initial denaturation followed by 40 cycles of $98^{\circ} \mathrm{C}$ for $1 \mathrm{~s}$ and $63^{\circ} \mathrm{C}$ for $15 \mathrm{~s}$. The total run time including ramp rates was $17 \mathrm{~min}$.

On-chip UF-qPCR assay (performed with an intercalating dye)

For the UF-qPCR assay, the same master mix was used as described before, but with increased primers and polymerase concentration: $20 \mu \mathrm{M}$ of sip primers and 1.68 units/ $\mu \mathrm{L}$ of KAPA2G HS fast polymerase (Kapa Biosystems, Wilmington, USA). Cycling conditions for UF-qPCR were $1 \mathrm{~min}$ initial denaturation at $95^{\circ} \mathrm{C}$, followed by 40 cycles of $98^{\circ} \mathrm{C}$ for $1 \mathrm{~s}$ and $63^{\circ} \mathrm{C}$ for $2 \mathrm{~s}$. The total run time including ramp rates is $4 \min 36 \mathrm{~s}$.

The GPCR mixtures were pipetted into the reaction chamber of the chip for both on-chip methods. 
Standard dilution series were prepared using freeze-dried DNA from S. agalactiae strain 2603 (BAA-611D-5, ATCC, Manassas, USA), which was purchased and resuspended in DEPC-treated water (Invitrogen, Carlsbad, USA). The genomic concentration (genome copies $/ \mathrm{mL}$ ) of the DNA was calculated using the measured DNA concentration (Nanodrop 2000, Thermo Scientific, Waltham, USA), the genome size and GC\% of the strain [19].

To obtain the same genome copy number per reaction, we took into account the difference in reaction volume between the benchtop reference qPCR assay and the on-chip assays. The latter one has a 4.17 times smaller reaction volume. Hence, for the calibration curves ranging from $10^{5}$ down to $10^{1}$ genome copies/reaction, we used 10 -fold serial dilutions from $5 \times 10^{7}$ down to $5 \times 10^{3}$ genome copies $/ \mathrm{mL}$ for the benchtop reference qPCR assay and 10-fold serial dilutions from $2.08 \times$ $10^{8}$ down to $2.08 \times 10^{4}$ genome copies $/ \mathrm{mL}$ for the on-chip assays.

To determine the limit of detection (LOD), a concentration of $6.24 \times 10^{3}$ genome copies $/ \mathrm{mL}$ of GBS DNA was tested 20 times in the UF-qPCR assay to assess the $95 \%$ probability of detecting 3 genome copies/reaction as defined by the MIQE guidelines [20]. Results were used to determine the LOD prediction interval.

\section{MELTING PROTOCOL}

After on-chip qPCR, the reaction mixtures were removed from the chip by aspiration, and melting curves were obtained using a LightCycler ${ }^{\odot 6}$ instrument (Roche, Base, Switzerland). Following protocol was used: $95^{\circ} \mathrm{C}$ for $10 \mathrm{~s}, 65^{\circ} \mathrm{C}$ for $60 \mathrm{~s}$ and then a continuous temperature increase, with a ramping rate of $0.2{ }^{\circ} \mathrm{C} / \mathrm{s}$ until $97^{\circ} \mathrm{C}$. Melting peaks were calculated with the LightCycler ${ }^{\odot} 96$ high resolution melting software.

\section{IMAGE ACQUISITION}

For the standard on-chip qPCR assay, a custom LabView script with automated filter wheel control was used to steer temperature cycling and to automate the image acquisition of the reaction chamber every cycle. 
For the UF-qPCR assay, a movie was recorded instead. Using the HClmage software (Hamamatsu, Hamamatsu city, Japan) the movie was decomposed into individual images at a rate of 4 frames per second, resulting in over 1100 images per on-chip qPCR reaction. The image with the highest mean grayscale value relative to the previous and subsequent image (i.e. acquired at $63^{\circ} \mathrm{C}$ ) was used to build the amplification curve.

\section{DATA ANALYSIS}

For the benchtop reference qPCR assay, raw data from the LightCycler ${ }^{\odot} 96$ was used to determine the $\mathrm{Ct}$ value by the crossing point method $\mathrm{Cy} 0$ in $\mathrm{R}$ [21] from the qpcR package [22].

For the on-chip assays, image analysis (using ImageJ software (NIH, Bethesda, USA)) and the Cy0 method were used to determine the Ct value. More details are available in the Supplemental methods.

\section{STATISTICAL ANALYSIS}

Concentrations were analyzed using the JMP software (SAS Institute, Cary, USA). Normality was tested using the Shapiro-Wilk test. Statistical hypothesis tests (regression, t-tests and equivalence tests) were considered significant for $p$ values $<0.05$.

Correlation of the benchtop reference qPCR and UF-qPCR results was determined using Spearman's rho value ( $\rho)$. The Bland-Altman method was used to visualize agreement between the two methods. Kappa values ( $\kappa$ ) were used to assess the proportion of agreement between both methods for assigning a positive or negative result.

\section{Results}

We selected a previously validated, hydrolysis probe-based qPCR assay for the quantification of $S$. agalactiae (group B streptococci, GBS) in clinical samples and used it with minor modifications [2]. As the benchtop reference method, this assay was run on a standard, commercial qPCR tool (LightCycler $\left.{ }^{\circledR} 96\right)$. Assay performance was verified by analyzing a 10 -fold dilution series of an $S$. agalactiae reference sample with known number of genome copies to establish a standard curve, 
showing an efficiency of $97 \%$ (Figure 2). Initial transfer of the assay from benchtop to microchip was performed with little adaptation of master mix composition (as defined by the manufacturer) and of cycling conditions, as an intermediate step in the process of speeding up the PCR. We refer to this method as our standard on-chip qPCR assay. The cycling time was decreased from $163.4 \mathrm{~s} /$ cycle to $23.7 \mathrm{~s} /$ cycle, resulting in a total reaction time of $17 \mathrm{~min}$ with a PCR efficiency of 99\% (Figure 2). To increase the reaction speed of the standard on-chip qPCR assay further, we increased primers and KAPA2G polymerase concentration by 4 and 10 times, respectively. Increasing the concentration of the KAPA2G polymerase mix (with and without hot-start) generated primer-dimers in the no template control (NTC). Increasing the KAPA2G hot-start polymerase and omitting the KAPA2G without hot-start polymerase in the $\mathrm{qPCR}$ reaction enabled us to decrease the assay time from an initial $163.4 \mathrm{~s} / \mathrm{cycle}$ (benchtop assay) to $4.8 \mathrm{~s} / \mathrm{cycle}$ (on-chip) as shown in Figure 1C. Including initial denaturation, we could perform qPCR within $4 \mathrm{~min} 36 \mathrm{~s}$ (see Supplemental video). The UF-qPCR on chip was completed before initial denaturation had finished on the benchtop tool (Figure 1C).

A calibration curve was used to determine the efficiency of the speeded up UF-qPCR assay as described for the standard on-chip qPCR. Figure 2 shows an efficiency of $94 \%$. Amplification curves are shown in Figure 3A. To verify the specificity of the amplification of the UF-qPCR, we performed melting curve analysis. Figure $3 \mathrm{~B}$ shows clear melting peaks with the expected value $\left(75^{\circ} \mathrm{C}\right.$ $\pm 0.42^{\circ} \mathrm{C}$ ) for each concentration. The limit of detection (LOD) for the UF-qPCR assay was determined. The theoretically lowest copy number that can be detected with $95 \%$ probability according to Poisson statistics is 3 copies per reaction, which corresponds to a concentration of $6.24 \times 10^{3}$ genome copies $/ \mathrm{mL}(\mathrm{Ct}=34.60)$. The UF-qPCR assay reaches this theoretical limit as 19 experiments out of 20 did showed amplification. The $95 \%$ prediction interval for the LOD ranges from $1.70 \times 10^{4}$ to $1.01 \times 10^{3}$ genome copies $/ \mathrm{mL}$, corresponding to Ct values from 33.12 to 37.23 . To assess clinical performance of the UF-qPCR assay, we quantified GBS in DNA extracted from 110 patient samples, comprising 27 vaginal, 28 rectal, 27 vaginorectal swabs and 28 LIM broth- 
enriched samples. The latter enriched samples were quantified by the chip PCR as containing between $6 \log 5$ and $4.7 \log 10 \mathrm{CFU} \mathrm{GBS} / \mathrm{mL}$. For sensitivity, samples with $\mathrm{Ct}$ values below 37.23 , were considered positive. For specificity, samples that showed no amplification (i.e. Ct values above 37.23) were identified as negative. The benchtop reference qPCR identified 100 out of 110 samples as positive. UF-qPCR detected GBS in 96 of the 100 positive samples, which corresponds to a $96 \%$ (Cl: $90 \%-98 \%)$ sensitivity. Seven out of 10 samples were correctly identified as negative by UFqPCR, which corresponds to a specificity of $70 \%$ (Cl: $40 \%-90 \%)$. Melting analysis, which was afterwards carried out off-chip, indicated the absence of a specific melting peak for all those 10 samples. Hence, if melting curve analysis were to be included on-chip, all true-negative samples would have been correctly identified, resulting in a specificity increase of $10 / 10$ or $100 \%$ (Cl: $72 \%-100 \%)$. At the same time, however, an incorrect melting peak was observed for 3 out of the 96 positive samples, which would lead to a decrease in sensitivity from $96 \%(96 / 100)$ to $93 \%$ (93/100 and $\mathrm{Cl}: 86 \%-96 \%)$.

We investigated the agreement between the UF-qPCR and the benchtop reference qPCR quantification results in various ways. First, correlation between UF-qPCR and the benchtop reference qPCR quantification results is highly significant as indicated by Spearman's $\rho=0.9445$, $p<0.001$. Second, as shown in Figure 4A, a simple linear regression between the two kinds of quantification results produced an intercept $(p=0.6066)$ and a slope $(p=0.4553)$ that are not significantly different from 0 and 1 , respectively. Third, we conducted an equivalence test for the two kinds of quantification results [23]: the two one-sided t-test procedure shows that the calculated $95 \%$ confidence interval of the mean difference between the two tests $(-0.1456$ to 0.1590$)$ falls within the equivalence region ( -0.5 to 0.5$)$. Fourth, we assessed the agreement between the two assays using a Bland-Altman plot. The Y-axis in that plot shows the log difference of the UF-qPCR assay and benchtop reference qPCR assay, while the X-axis shows the average log of the two assays (Figure 4B). The bias between the two assays is 0.0047 , whereas $81 \%$ and $99 \%$ of the 
results lie between -0.5 and $0.5 \log$ genome copies $/ \mathrm{mL}$ and -1.0 and $1.0 \log$ genome copies $/ \mathrm{mL}$ [24], respectively, indicating strong agreement between the two assays.

When performing a qualitative analysis of the UF-qPCR assay and the benchtop assay by only looking at positive and negative observations, the Kappa coefficient $\kappa$ indicates an agreement between the UF-qPCR and benchtop reference qPCR assay results of 0.74 with and 0.63 without melting curve analysis. The significance for both these values is $p<0.0001$. 


\section{Discussion}

The screening for GBS during pregnancy is part of a routine check-up performed at 35-37 weeks of gestation. GBS present in rectum and/or vagina can be transmitted to the newborn during childbirth, potentially leading to pneumonia, meningitis and/or death of the neonate. This screening strategy is aimed at the identification of the $10-30 \%$ of women who are vaginal and/or rectal GBS carriers who should receive intrapartum antibiotic prophylaxis. This antepartum test for GBS does not accurately predict genital tract colonization at the time of labor [25]. As a consequence, women found negative at screening but positive at labor will be withheld treatment and, vice versa, women who convert from positive to negative will receive unnecessary treatment. Furthermore, women who deliver preterm or enter late into prenatal care do not get the chance to be screened at 35-37 weeks of gestation, and therefore, their GBS status is not known at the time of labor [3,25-27]. This indicates the need for a rapid and sensitive test that allows intrapartum detection of GBS. Hence, the UF-qPCR-based test described here could be the catalyst for the development of a point-ofcare assay, not only to limit the number of false-negative test results, but also to decrease the overuse of antibiotics. A regular QPCR assay takes commonly at least one hour whereas the onchip UF-qPCR assay described here is completed within 4 min $36 \mathrm{~s}$. The speed improvement was only possible after increasing polymerase and primers concentration. For the polymerase, we used a hot-start polymerase instead of a combined mixture of non-hot-start and hot-start polymerases to avoid primer-dimer formation. Also, an intercalating dye instead of a Taqman probe was used, due to the fact that at present the fastest polymerases on the market do not have 5' exonuclease activity [28]. Importantly, the decrease in runtime did not affect the efficiency of the qPCR assay.

Statistical analysis by means of Spearman's $\rho$, and linear regression show that there is strong correspondence between the results obtained using the on-chip UF-qPCR assay and the benchtop reference qPCR (Figure 4A). In addition, the two one-sided t-test indicates statistical equivalence of the two assays and a Bland-Altman plot (Figure 4B) shows that the quantification is in good agreement [24]. Finally, for the qualitative comparison, the Kappa coefficient $\kappa$ also indicates a 
strong agreement by far exceeding any level of agreement that can be explained by chance alone [29].

Use of melting curve analysis after UF-qPCR increased assay specificity, but decreased assay sensitivity. Three samples were identified correctly as positive, i.e. in agreement with the benchtop reference qPCR method, but these samples did not show a correct melting peak after UF-qPCR and should therefore be considered negative when taking into account melting curve data. For two of these samples, concentrations were indeed lower than the theoretical LOD of the UF-qPCR assay due to the fact that the chip reaction volume is 4.17 times smaller than that of the benchtop reference qPCR assay. This can only be improved by increasing reaction cavity volume and, hence, chip size. On the other hand, specificity increased from $70 \%$ to $100 \%$ by including melting analysis. The three samples that were false-positive could be corrected and considered as negative by taking the melting curve data into account. Although adding melting curve analysis to the UF-qPCR will evidently increase the run time of the assay, recent work by Pryor and colleagues indicates that it is possible to perform high resolution melting within 1 second [30], suggesting that the increase is negligible.

As mentioned above, the cavity volume of the UF-qPCR described here is more than four times smaller than that of the benchtop reference qPCR assay, which is an advantage for sample consumption. The reagent cost was, however, not reduced as we needed to increase the amount of polymerase to speed up the qPCR reaction. We only tested three different polymerase concentrations, it could be useful to do a more thorough screening of the amount of polymerase to be used. The chips used during the experiments were also relatively large and contained multiple cavities. In the future, we envision the use of smaller, single-cavity, single-use chips as the chip cost scales with its size.

The development of UF-qPCR, possible by combining well-established semiconductor chip technology and molecular biology tools, can speed up the commercialization of fast commercial qPCR tools, which are currently slowly entering the market, for example the xxpress from BJS 
Biotechnologies (40-cycle qPCR in 10 min: Xxpress from BJS Biotechnologies) and the genechecker from Genesystem (30-cycle qPCR in 12 min: Genechecker from Genesystem Co., Ltd). Ultimately, however, the goal is to combine fast qPCR with integrated sample preparation, realizing while-you-wait testing in under $15 \mathrm{~min}$. By combining techniques that perform nucleic acid extraction in under $10 \mathrm{~min}$ [31], and UF-qPCR chips that perform amplification in under 5 minutes, we are close to reaching this goal. The fastest currently available commercial solution to offer a DNA-based sample-to-answer result for GBS testing is the GeneXpert from Cepheid, which requires 50 min (Xpert $^{\circledast}$ GBS from Cepheid). UF-qPCR could also outperform antibody-based approaches such as lateral flow immunoassays, both in terms of speed and sensitivity $[32,33]$.

In conclusion, our research shows that it is possible to accurately and efficiently quantify nucleic acids using qPCR within 5 min. We expect that combining fast DNA extraction, UF-qPCR and fast high-resolution melting, producing a complete sample-to-result device that requires less than 15 min from start to finish, may soon be feasible. We hope that our work will contribute to the development of these fast and accurate diagnostic devices for use at the point of care. 


\section{Acknowledgements}

We would like to thank Gabrielle Woronoff for her help in creating the Supplemental video, and Leen Van Simaey and Senne Cornelis for preparing and transporting the clinical samples.

We declare that we have no conflict of interest. 


\section{References}

[1] S. Schrag, R. Gorwitz, K. Fultz-Butts, A. Schuchat, Prevention of perinatal group B streptococcal disease. Revised guidelines from CDC., MMWR. Recomm. Reports Morb. Mortal. Wkly. Report. Recomm. Reports. $51 \quad$ (2002) 1-22. http://www.ncbi.nlm.nih.gov/pubmed/12211284 (accessed July 31, 2018).

[2] N.A. El Aila, I. Tency, G. Claeys, H. Verstraelen, P. Deschaght, E. Decat, G. Lopes dos Santos Santiago, P. Cools, M. Temmerman, M. Vaneechoutte, Comparison of culture with two different qPCR assays for detection of rectovaginal carriage of Streptococcus agalactiae (group B streptococci) in pregnant women, Res. Microbiol. 162 (2011) 499-505. http://dx.doi.org/10.1016/j.resmic.2011.04.001.

[3] J.R. Verani, L. McGee, S.J. Schrag, Division of Bacterial Diseases, National Center for Immunization and Respiratory Diseases, Centers for Disease Control and Prevention (CDC), Prevention of perinatal group B streptococcal disease--revised guidelines from CDC, 2010., MMWR. Recomm. Reports Morb. Mortal. Wkly. Report. Recomm. Reports. 59 (2010) 1-36. http://www.ncbi.nlm.nih.gov/pubmed/21088663 (accessed July 31, 2018).

[4] S.A. Thatcher, DNA/RNA preparation for molecular detection, Clin. Chem. 61 (2015) 89-99.

[5] J.S. Farrar, C.T. Wittwer, Extreme PCR: efficient and specific DNA amplification in 15-60 seconds, Clin. Chem. 61 (2015) 145-153.

[6] S. Yang, R.E. Rothman, PCR-based diagnostics for infectious diseases: Uses, limitations, and future applications in acute-care settings, Lancet Infect. Dis. 4 (2004) 337-348.

[7] J.F. Mackay, Taking it to the extreme: PCR at wittwerspeed, Clin. Chem. 61 (2015) 4-5.

[8] S. Brunklaus, T.E. Hansen-Hagge, J. Erwes, J. Höth, M. Jung, D. Latta, X. Strobach, C. Winkler, M. Ritzi-Lehnert, K.S. Drese, Fast nucleic acid amplification for integration in pointof-care applications, Electrophoresis. 33 (2012) 3222-3228.

[9] D. Moschou, N. Vourdas, G. Kokkoris, G. Papadakis, J. Parthenios, S. Chatzandroulis, A. Tserepi, All-plastic, low-power, disposable, continuous-flow PCR chip with integrated 
microheaters for rapid DNA amplification, Sensors Actuators, B Chem. 199 (2014) 470-478.

[10] M.U. Kopp, A.J. De Mello, A. Manz, Chemical amplification: continuous-flow PCR on a chip, Science (80-. ). 280 (1998) 1046-1048.

[11] M. Hashimoto, P.-C. Chen, M.W. Mitchell, D.E. Nikitopoulos, S. a Soper, M.C. Murphy, Rapid PCR in a continuous flow device., Lab Chip. 4 (2004) 638-45.

[12] Y. Fuchiwaki, H. Nagai, M. Saito, E. Tamiya, Ultra-rapid flow-through polymerase chain reaction microfluidics using vapor pressure, Biosens. Bioelectron. 27 (2011) 88-94.

[13] P. Neuzil, C. Zhang, J. Pipper, S. Oh, L. Zhuo, Ultra fast miniaturized real-time PCR: 40 cycles in less than six minutes, Nucleic Acids Res. 34 (2006) 9.

[14] H. Kim, S. Dixit, C.J. Green, G.W. Faris, Nanodroplet real-time PCR system with laser assisted heating, Opt. Express. 17 (2008) 218.

[15] A.F.R. Huhmer, J.P. Landers, Noncontact infrared-mediated thermocycling for effective polymerase chain reaction amplification of DNA in nanoliter volumes, Anal. Chem. 72 (2000) $5507-5512$.

[16] E.K. Wheeler, C.A. Hara, J. Frank, J. Deotte, S.B. Hall, W. Benett, C. Spadaccini, N.R. Beer, Under-three minute PCR: Probing the limits of fast amplification, Analyst. 136 (2011) 3707.

[17] H. Terazono, H. Takei, A. Hattori, K. Yasuda, Development of a high-speed real-time polymerase chain reaction system using a circulating water-based rapid heat-exchange, Jpn. J. Appl. Phys. 49 (2010) 06GM05.

[18] B. Majeed, B. Jones, D.S. Tezcan, N. Tutunjyan, L. Haspeslagh, S. Peeters, P. Fiorini, M.O. De Beeck, C. Van Hoof, M. Hiraoka, H. Tanaka, I. Yamashita, Silicon based system for singlenucleotide-polymorphism detection: Chip fabrication and thermal characterization of polymerase chain reaction microchamber, Jpn. J. Appl. Phys. 51 (2012) 1-9.

[19] H. Tettelin, V. Masignani, M.J. Cieslewicz, J.A. Eisen, S. Peterson, M.R. Wessels, I.T. Paulsen, K.E. Nelson, I. Margarit, T.D. Read, L.C. Madoff, A.M. Wolf, M.J. Beanan, L.M. Brinkac, S.C. Daugherty, R.T. DeBoy, A.S. Durkin, J.F. Kolonay, R. Madupu, M.R. Lewis, D. 
Radune, N.B. Fedorova, D. Scanlan, H. Khouri, S. Mulligan, H.A. Carty, R.T. Cline, S.E. Van Aken, J. Gill, M. Scarselli, M. Mora, E.T. Iacobini, C. Brettoni, G. Galli, M. Mariani, F. Vegni, D. Maione, D. Rinaudo, R. Rappuoli, J.L. Telford, D.L. Kasper, G. Grandi, C.M. Fraser, Complete genome sequence and comparative genomic analysis of an emerging human pathogen, serotype V Streptococcus agalactiae, Proc Natl Acad Sci U S A. 99 (2002) 1239112396.

[20] S.A. Bustin, V. Benes, J.A. Garson, J. Hellemans, J. Huggett, M. Kubista, R. Mueller, T. Nolan, M.W. Pfaffl, G.L. Shipley, J. Vandesompele, C.T. Wittwer, The MIQE guidelines: Minimum information for publication of quantitative real-time PCR experiments, Clin. Chem. 55 (2009) 611-622.

[21] M. Guescini, D. Sisti, M.B.L. Rocchi, L. Stocchi, V. Stocchi, A new real-time PCR method to overcome significant quantitative inaccuracy due to slight amplification inhibition, BMC Bioinformatics. 9 (2008) 1-12.

[22] C. Ritz, A.N. Spiess, qpcR: An R package for sigmoidal model selection in quantitative realtime polymerase chain reaction analysis, Bioinformatics. 24 (2008) 1549-1551.

[23] P. Goos, D. Meintrup, Statistics with JMP: Hypothesis Tests, ANOVA and regression, John Wiley and sons, 2016.

[24] J.M. Bland, D.G. Altman, Measuring agreement in method comparison studies, Stat. Methods Med. Res. 8 (1999) 135-160.

[25] B.C. Young, L.E. Dodge, M. Gupta, J.S. Rhee, M.R. Hacker, Evaluation of a rapid, real-time intrapartum group B streptococcus assay, Am. J. Obstet. Gynecol. 205 (2011) 372-373.

[26] M.J. Alfa, S. Sepehri, P. De Gagne, M. Helawa, G. Sandhu, G.K.M. Harding, Real-time PCR assay provides reliable assessment of intrapartum carriage of group B Streptococcus, J. Clin. Microbiol. 48 (2010) 3095-3099.

[27] C.A. Haberland, W.E. Benitz, G.D. Sanders, J.B. Pietzsch, S. Yamada, L. Nguyen, A.M. Garber, Perinatal screening for group B streptococci: Cost-benefit analysis of rapid 
polymerase chain reaction, Pediatrics. 110 (2002) 471-480.

[28] J.L. Montgomery, N. Rejali, C.T. Wittwer, Stopped-flow DNA polymerase assay by continuous monitoring of dNTP incorporation by fluorescence, Anal. Biochem. 441 (2013) 133-139.

[29] A.J. Viera, J.M. Garrett, Understanding Interobserver Agreement: The Kappa Statistic, Fam. Med. 37 (2005) 360-363.

[30] R.J. Pryor, J.T. Myrick, R.A. Palais, S.O. Sundberg, J.Y. Paek, C.T. Wittwer, I.T. Knight, Highspeed melting analysis: The effect of melting rate on small amplicon microfluidic genotyping, Clin. Chem. 63 (2017) 1624-1632.

[31] Y. Zou, M.G. Mason, Y. Wang, E. Wee, C. Turni, P.J. Blackall, M. Trau, J.R. Botella, Nucleic acid purification from plants, animals and microbes in under 30 seconds, PLOS Biol. 15 (2017) e2003916.

[32] M. Kong, J.H. Shin, S. Heu, J.K. Park, S. Ryu, Lateral flow assay-based bacterial detection using engineered cell wall binding domains of a phage endolysin, Biosens. Bioelectron. 96 (2017) 173-177.

[33] P. López-Soriano, P. Noguera, M.T. Gorris, R. Puchades, Á. Maquieira, E. Marco-Noales, M.M. López, Lateral flow immunoassay for on-site detection of Xanthomonas arboricola pv. pruni in symptomatic field samples, PLoS One. 12 (2017) 1-13. 


\section{Figures}

Figure $1 \mathrm{~A}$ silicon-based chip was used to perform UF-qPCR. A) Photograph of the silicon chip with four reaction chambers. Each reaction chamber is enclosed by insulating trenches. B) Mask design of the silicon chip with an insulated reactor width of $3600 \mu \mathrm{m}$. C) Temperature versus time trace of benchtop reference qPCR assay versus UF-qPCR. The inset shows that the UF-qPCR assay is completed before initial denaturation has ended on the benchtop tool.

Figure 2 Calibration curves for the GBS qPCR performed with the benchtop reference qPCR assay (LightCycler $\left.{ }^{\odot 96}\right)$ and with two on-chip assays. The benchtop reference qPCR assay $(\mathrm{N}=7)$ represented by blue squares and the fitted line shown as a blue dashed-and-dotted line, standard on-chip qPCR assay of $17 \mathrm{~min}(\mathrm{~N}=2)$ represented in fuchsia and the fitted line shown as a fuchsia dotted line and UF-qPCR assay of $4 \min 36 \mathrm{~s}(\mathrm{~N} \geq 4)$ represented in green triangles with the fitted line shown as a green full line, with efficiencies of $97 \%, 99 \%$ and $94 \%$ respectively.

Figure 3 Amplification $(A)$ and melting curves $(B)$ for a 10-fold dilution series of a reference sample of $S$. agalactiae genomic DNA, with concentrations spanning 5 log units and the no template control (NTC) performed with the UF-qPCR assay. A) Fluorescence versus cycle number. B) First derivative plots of the melting curves showing the specific melting peaks.

Figure 4 Comparison of quantification results for GBS in the clinical samples as obtained using benchtop reference qPCR assay (X-axis) versus UF-qPCR (Y-axis). Statistical analysis of the patient samples using A) regression analysis, which shows that the correlation between UF-qPCR and benchtop reference qPCR assay quantification results is highly significant and B) Bland-Altman plot, which shows strong agreement between the UF-qPCR and benchtop reference qPCR assay. 


\section{Figure 1}

A

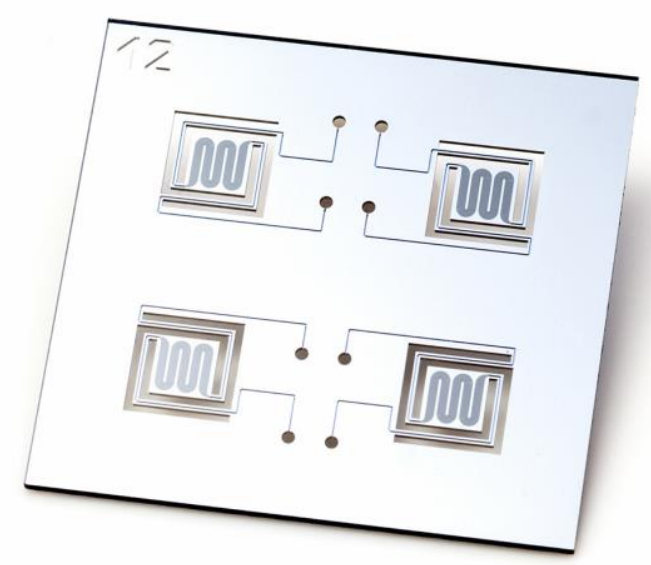

B

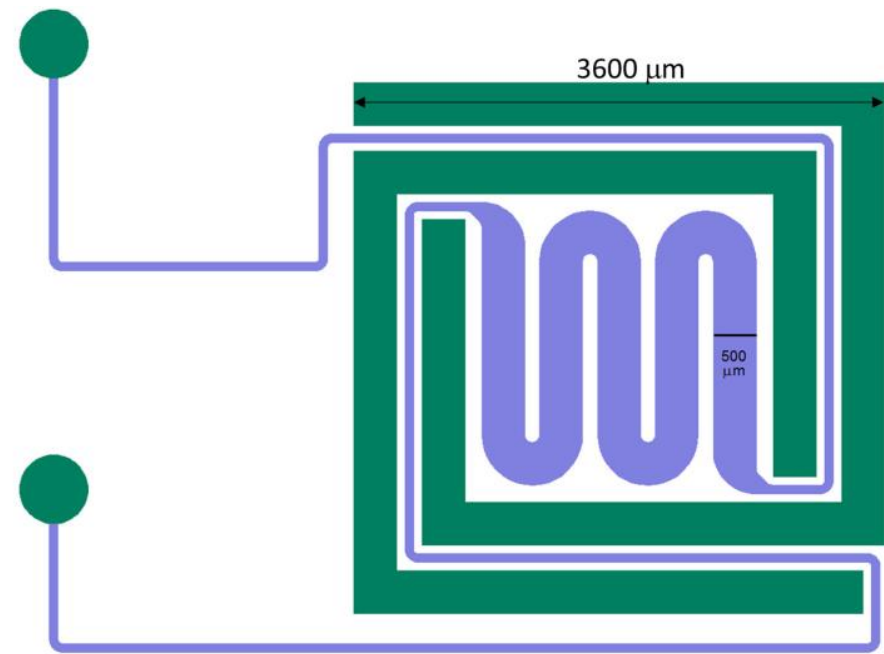

C Benchtop reference qPCR UF-qPCR
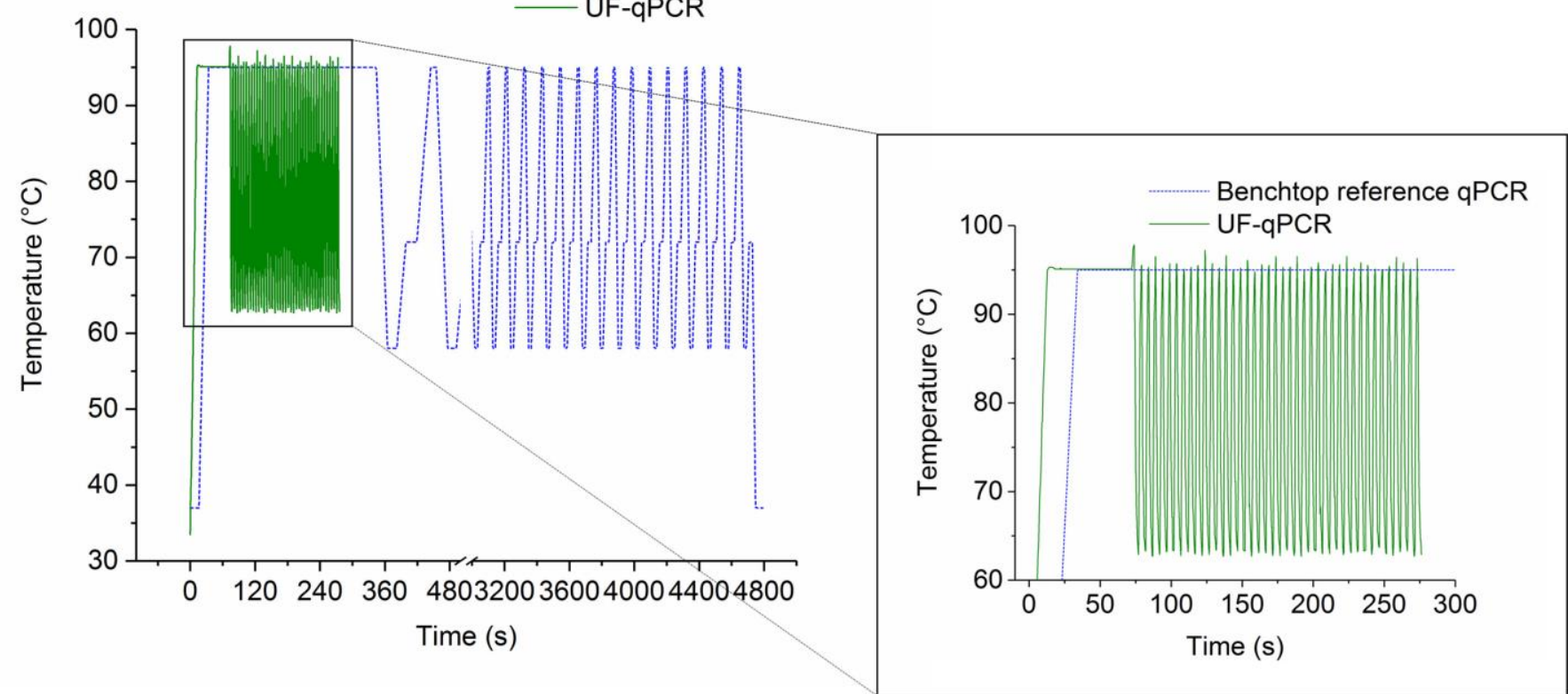
Figure 2 


\section{Figure 3}

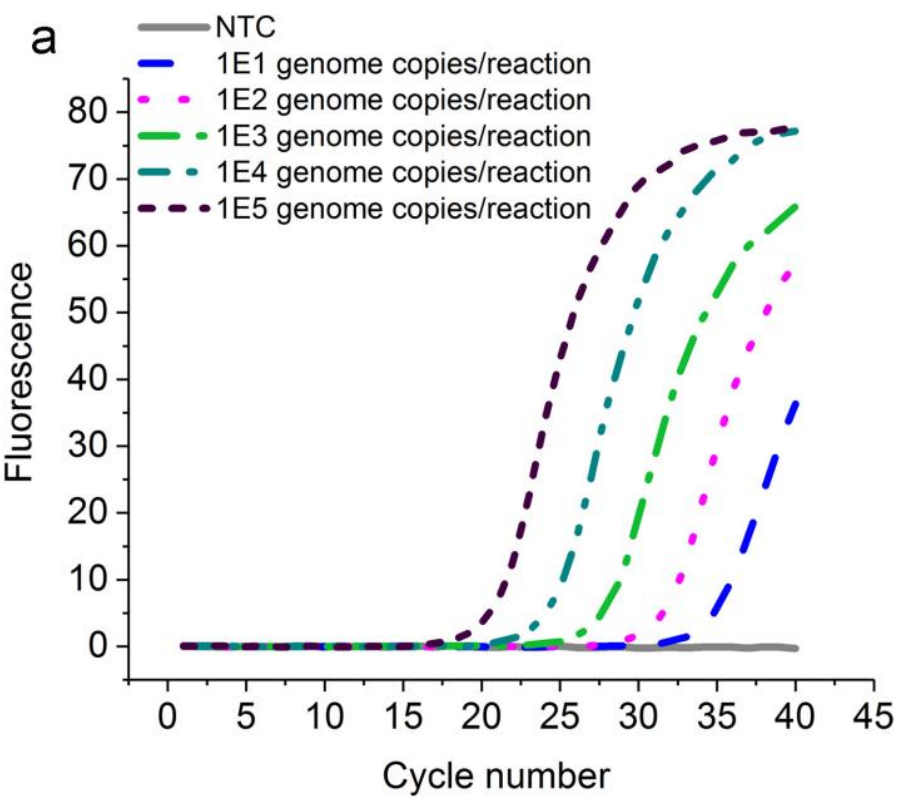

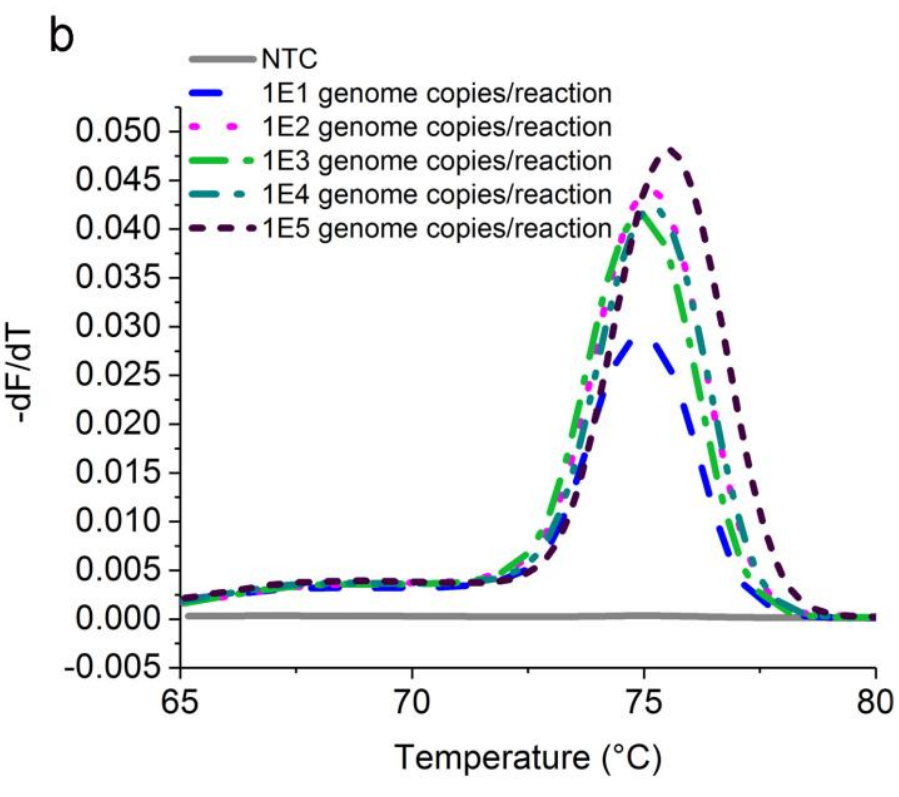




\section{Figure 4}

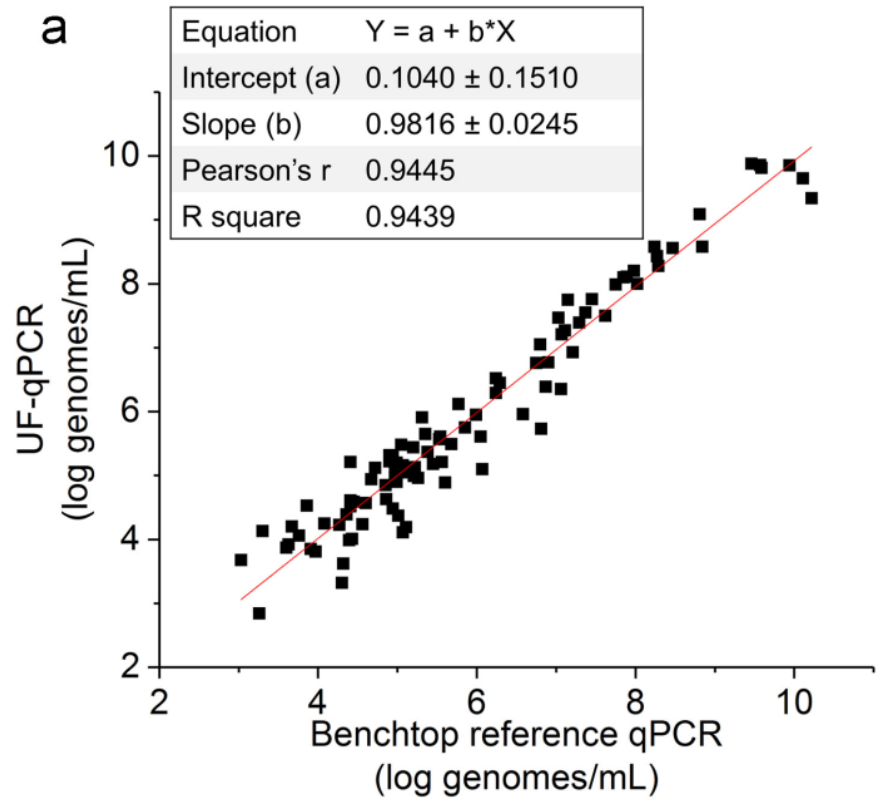

b

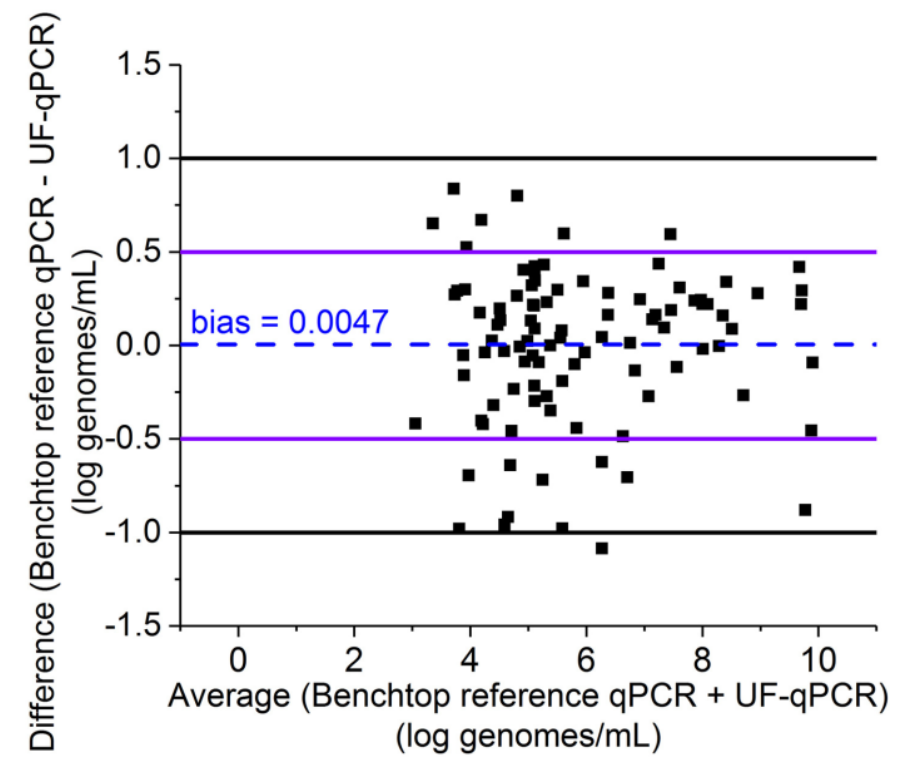

